\title{
Frailty as a Predictor of Nursing Home Placement among Community-Dwelling Older Adults: A Systematic Review and Meta-analysis
}

\author{
Gotaro Kojima, $\mathrm{MD}^{1}$ \\ ${ }^{1}$ Japan Green Medical Centre, London, United Kingdom. \\ Corresponding Author: \\ Gotaro Kojima, MD \\ Japan Green Medical Centre \\ 10 Throgmorton Avenue, \\ London, EC2N 2DL, United Kingdom \\ Email: gotarokojima@yahoo.co.jp \\ Phone: +44(0)20-7330-1750 \\ Fax: +44(0)20-7330-1751
}

\begin{abstract}
Background and Purpose: Frailty has been recognized as a medical syndrome characterized by decreased physiological reserve and vulnerability to poor resolution of homeostasis.

Frailty is common in nursing homes and as many as $90 \%$ of nursing home patients are frail or prefrail. Previous studies have examined frailty as a risk factor for nursing home placement and yielded inconsistent results. The current study aims to systematically review the literature and to conduct a meta-analysis to combine the risk measures to provide the evidence on frailty as a predictor of nursing home placement among community-dwelling older adults. Methods: A systematic literature search was performed in July 2015 using 6 databases (Scopus, Embase, CINAHL Plus, MEDLINE, PsycINFO, and the Cochrane Library). Any cohort studies examining associations between frailty and risk of subsequent nursing home placement among community-dwelling older adults published from 2000 to July 2015 were potentially eligible. The numbers of those who were admitted to nursing homes and who were not, according to frailty categories, were used to calculate pooled OR using fixed-effect models. The included studies were assessed for heterogeneity, methodological quality, and publication bias. The systematic literature search and hand-search identified 885 potentially relevant studies, among which 5 studies including 3,528 community-dwelling older adults were selected for this review. Results: Meta-analyses were performed using data from these studies and showed that both frailty and prefrailty significantly predicted nursing home placement ( 5 studies: pooled $\mathrm{OR}=5.58,95 \% \mathrm{CI}=2.94-10.60, \mathrm{p}<0.00001 ; 3$ studies: pooled $\mathrm{OR}=3.26,95 \% \mathrm{CI}=1.21-8.78, \mathrm{p}=0.02$, respectively). Heterogeneity across the studies was low or moderate and there was no evidence of publication bias. Discussion: Frailty generally progresses but can also be potentially modified by appropriate interventions such as physical exercise. Evidence especially has shown that aerobic and resistance exercises improve frailty components. Furthermore, a multifactorial interdisciplinary intervention, including tailored exercise programs, has shown to decrease prevalence of frailty among frail communitydwelling older adults. It is noteworthy that adherence to the exercise programs was high and adverse events were not reported in most of the trials. These findings suggest that physical exercise can potentially prevent or reverse frailty and may lead to decreased risks of nursing home placement in older adults. Conclusions: This systematic review and meta-analysis study is the first to report evidence that both frailty and prefrailty are significant predictors of nursing home placement among community-dwelling older adults.
\end{abstract}

Keywords: Frailty; Nursing home placement; Institutionalization; Community-dwelling older adults; Systematic review; Meta-analysis. 


\section{INTRODUCTION}

Older adults are at high risk of nursing home placement. In developed countries $1.5-8 \%$ of older adults aged 65 or more are living in nursing homes. ${ }^{1}$ Most older adults prefer to stay in their familiar home environment rather than transfer to a nursing home because the transition to nursing home can cause loss of autonomy and independence, poor quality of life, and negative psychological impacts. ${ }^{2,3}$ In addition, the cost of nursing home placement is substantial and imposes a huge burden on patients and their family as well as heathcare systems, stretching limited healthcare resources. ${ }^{4}$ With a growing number of older adults worldwide, there have been increasing implications to avoid or delay nursing home placement and extend quality life in old age.

Determining modifiable risk factors of nursing home placement is of interest to older adults, their families, and all related parties, including clinicians, researchers, and policymakers. High-risk individuals can be identified and targeted for interventions to prevent or delay admission to the nursing home. Moreover, those at high risk can be educated to prepare themselves to make decisions in advance and be guided in their future financial planning, leading to a better overall quality of life in old age. Among various risk factors associated with nursing home placement reported in the literature, functional disability, such as difficulty in performing basic activities of daily living, has been extensively studied and almost uniformly shown to be a strong predictor. ${ }^{5}$ Once disability is fully developed, it may be difficult to modify. An old but recently reconceptualized entity, frailty, has been gaining the attention of recent research. Frailty was once used synonymously with disability as these 2 often coexist in older adults, with overlapping features. However, frailty is now considered as a distinct state with a biological basis and may be a pre-disability state. ${ }^{6}$ Frailty has been described as a medical syndrome characterized by decreased physiological reserve in multiple systems and vulnerability to poor resolution of homeostasis after internal or external stressors due to accumulated age-related health deficits. ${ }^{6-8}$ The most frequently used frailty definition is the phenotype criteria proposed by Fried et al., using the Cardiovascular Health Study (CHS) data. ${ }^{6}$ They defined frailty categories based on 5 physical components: weight loss, exhaustion, weakness, slow walking speed, and low physical activity. ${ }^{6}$ An individual is classified as robust, prefrail, and frail when they meet $0,1-2$, and 3 5 components. ${ }^{6}$ Frailty is associated with multiple adverse health outcomes, including disability, falls, hospitalization, dementia, poor quality of life, and mortality. ${ }^{6-14}$ Prevalence of frailty among community-dwelling older adults is approximately $10 \%,{ }^{15}$ while frailty is highly prevalent in nursing homes, with as many as 50\% of nursing home patients being frail. ${ }^{16}$ Given the dynamic nature of frailty, which can progress or improve dramatically, ${ }^{17}$ and its potential to be a precursor of disability, ${ }^{6}$ frailty may be a promising target in order to reduce nursing home placement risks in older adults. Although frailty can easily be expected to be a cause of nursing home placement, only a limited amount of evidence on frailty as a predictor of nursing home placement exists in the literature. Several prospective studies have examined associations between baseline frailty and a subsequent risk of nursing home admission, with inconsistent results. ${ }^{18-24}$

One previous systematic review paper searched for studies published in 1990-2010, examining associations between frailty and nursing home placement in general elderly populations. ${ }^{25}$ The review paper identified only 2 studies, ${ }^{23,24}$ both of which showed frailty was a significant predictor of nursing home placement, but did not perform a meta-analysis to show pooled evidence. ${ }^{25}$ Since this review, an increasing number of relevant studies have been expected be published, ${ }^{18-21}$ as this research field has been making remarkable advances. Thus, the current study aimed (1) to systematically review the literature for prospective 
studies investigating frailty as a risk factor for nursing home placement among communitydwelling older adults and (2) to conduct a meta-analysis to combine the risk measures to provide pooled evidence.

\section{METHOD}

A systematic search of the literature was conducted by 1 geriatric clinician researcher (GK) with experience in outpatient and nursing home settings in July 2015 according to a protocol developed within the scope of the Meta-analysis of Observational Studies in Epidemiology (MOOSE) statements. ${ }^{26}$ Any cohort studies examining associations between frailty and a subsequent nursing home placement risk among community-dwelling older adults, published from 2000 through July 2015, were searched for without language restriction using 6 electronic databases (Scopus, Embase, CINAHL Plus, MEDLINE, PsycINFO, and the Cochrane Library). The search terms used were "Institutionalization (Medical Subject Heading (MeSH))", "Patient admission (MeSH)", "Facility admission (MeSH)", "institutionalization*", "institutionalisation*", "nursing home placement*", "nursing home admission*", "Frailty syndrome (MeSH)", and "frailty". Bibliographies of the relevant articles were also hand-searched. An expert in this field (Dr Yu Taniguchi) was consulted for any additional articles.

Inclusion criteria were: (1) original articles; (2) involving non-institutionalized older adults aged 65 years or older living in the community; (3) longitudinally examining a risk of nursing home placement according to baseline frailty status defined by validated criteria or its modified versions; and (4) providing odds ratio (OR) as a risk measure or data sufficient enough to calculate OR. Authors of potentially eligible studies were contacted for sharing data. Exclusion criteria were: (1) defining frailty using surrogate measures, such as morbidity or walking speed; (2) using a non-general population, such as patients with heart failure or dementia; or (3) using a continuous index to grade frailty status without categorizing frailty status. If the same cohort was used by multiple eligible studies, the study with the largest number of participants was included. All eligible studies were assessed for methodological quality using the Newcastle-Ottawa scale for cohort studies. ${ }^{27}$ This scale was chosen because it was developed by international experts to assess the quality of nonrandomized studies for meta-analysis. ${ }^{27}$ It consists of 9 items to assess selection, compatibility, and outcome domains of cohort studies. ${ }^{27}$ In this review, a study was considered to have adequate methodological quality to be included in the meta-analysis if 5 or more out of 9 criteria were met. Data extracted were first author, study cohort name, publication year, location (country), sample size, proportion of female individuals, age (mean, range, or age criterion for inclusion), frailty criteria, effect measure, and follow-up period.

A flow diagram of the systematic literature search and selection process, along with the number of studies at each stage are shown in Figure 1. Of these 885 studies identified, 5 were included for this review. All 5 studies were considered to have adequate methodological quality (mean number of criteria met=5.8, range=5-7) (Table 1).

\section{Statistical Analysis}

The number of participants who were admitted to nursing homes and who were not according to frailty categories (frail, prefrail, and robust) were collected from the included studies and used to calculate pooled OR using the Mantel-Haenszel method. The heterogeneity among the included studies was assessed using Cochran's Q statistic and the magnitude of the heterogeneity was assessed using the $\mathrm{I}^{2}$ statistic. $\mathrm{I}^{2}$ values of $25 \%, 50 \%$, and $75 \%$ were considered as low, moderate, and high heterogeneity, respectively. ${ }^{28} \mathrm{~A}$ fixed-effects model 
was used if the heterogeneity was low to moderate and a random-effects model was used if the heterogeneity was high. The number of participants who were admitted to nursing homes and who were not according to baseline frailty categories (frail, prefrail, and robust) from the 5 included studies were used for the meta-analysis. Pooled OR was calculated using fixedeffects models based on low to moderate heterogeneity across the studies ( 5 studies: $p=0.11$, $\mathrm{I}^{2}=46 \%$ for frailty; 3 studies: $\mathrm{p}=0.60, \mathrm{I}^{2}=0 \%$ for prefrailty). Publication bias was assessed using Begg-Mazumdar's and Egger's tests and visually inspecting funnel plots. All analyses were performed using Review Manager 5 (version 5.2, The Cochrane Collaboration, Copenhagen, Denmark) and StatsDirect (version 2.8, StatsDirect, Cheshire, UK).

\section{RESULTS}

\section{Study Characteristics}

Table 1 presents characteristics of the 5 included studies encompassing 3,528 communitydwelling older adults. ${ }^{18-21,23}$ Four studies were from Europe ${ }^{18-21}$ (Italy, Portugal, and Netherlands) and 1 from the United States. ${ }^{23}$ All studies included relatively small numbers of participants, ranging from $95^{21}$ to $1,679 .{ }^{19}$ One study included only women. ${ }^{23}$ Not all studies presented mean age, but the participants were mostly aged 70 or above. Various criteria were used to define frailty, including the CHS criteria, ${ }^{21,23}$ the Study of Osteoporotic Fractures (SOF) frailty index, ${ }^{18,20}$ the Conselice Study of Brain Aging (CSBA) index,${ }^{20}$ and the Frailty Index (FI). ${ }^{19}$ Bilotta et al. used the SOF frailty index, which consists of 3 components, and defined frail, prefrail, and robust as having 2 or more, 1 , and 0 components, respectively. ${ }^{18}$ One study using the $\mathrm{FI}^{19}$ divided the cohort into tertiles, therefore, the highest, middle, and lowest tertiles were treated as frail, prefrail, and robust groups, respectively, in the current review. Two studies ${ }^{20,21}$ dichotomized the cohort into frail and non-frail and 3 studies ${ }^{18,19,23}$ divided the cohort into frail, prefrail, and robust. Among the 5 included studies, $3^{19,20,23}$ showed that frailty was associated with significantly higher risks of nursing home admission while the other 2 studies ${ }^{18,21}$ did not. Three studies examined the risks of nursing home placement according to prefrail and all showed nonsignificant results. ${ }^{18,19,23}$ Follow-up periods ranged from 10 months ${ }^{21}$ up to 4 years,${ }^{20}$ with the mean of 2.2 years.

\section{Frailty as a Predictor of Nursing Home Placement}

Frailty and prefrailty were significantly associated with a higher risk of nursing home placement compared with non-frailty (5 studies: pooled $\mathrm{OR}=5.58,95 \% \mathrm{CI}=2.94-10.60$, $\mathrm{p}<0.00001 ; 3$ studies: pooled $\mathrm{OR}=3.26,95 \% \mathrm{CI}=1.21-8.78, \mathrm{p}=0.02$, respectively) (Figure 2)

\section{Publication Bias Assessment}

Figures $\mathbf{3} \mathbf{A}$ and $\mathbf{B}$ are funnel plots for studies examining a nursing home placement risk based on frailty and prefrailty, respectively. No apparent asymmetry suggestive of publication bias was observed. Begg-Mazumdar's and Egger's tests showed no evidence of publication bias for studies of frailty (both $\mathrm{p}=0.82$ ). These tests could not assess the 3 studies for prefrailty due to the small number of studies.

\section{DISCUSSION}

Both frailty and prefrailty have been demonstrated to be a significant predictor of nursing home placement based on the pooled data of 5 studies included in this systematic review and meta-analysis.

Among the included 5 studies, 3 defined frailty based on physical components using the CHS criteria ${ }^{21,23}$ or the SOF frailty index. ${ }^{18}$ The physical components used included unintentional weight loss, self-reported exhaustion/low energy, weakness (hand grip), slow walking speed, 
low physical activity, and inability to rise from a chair. ${ }^{6,29}$ Meanwhile, 2 studies employed multidimensional frailty tools, the $\mathrm{FI}^{19}$ and the CSBA index, ${ }^{20}$ which encompass cognitive, psychological, and social components in addition to physical components to conceptualize frailty. Although the CHS criteria, focusing on the physical dimension, has been most commonly used in the literature, the multidimensional approaches to define frailty have recently been more debated. ${ }^{30-32}$ The multidimensional criteria were compared with the CHS criteria and shown to have better discriminating abilities than the CHS criteria in some studies. ${ }^{33,34}$ In the current review, a subgroup analysis according to frailty criteria showed no significant nursing home placement risk difference ( $\mathrm{p}$ for group difference $=0.79$ ) between 2 studies ${ }^{19,20}$ using multidimensional frailty criteria (pooled $\mathrm{OR}=5.99,95 \% \mathrm{CI}=2.60-13.81$, $\mathrm{p}<0.0001$ ) and 3 studies $^{18,21,23}$ using physical frailty criteria (pooled $\mathrm{OR}=5.00,95 \% \mathrm{CI}=1.84$ $13.59, \mathrm{p}=0.03)$.

Frail and prefrail older adults are approximately 5 times and 3 times more likely to be institutionalized, respectively, compared with those who are non-frail. The mechanisms of how frailty predisposes older adults to being institutionalized are not clear, but may be multifactorial since the reasons for nursing home placement are often complex and are subject to social and cultural circumstances. One possible explanation is disability. Frail older adults are at a high risk of developing disability, ${ }^{35}$ which can be the direct cause of nursing home placement. One of the included studies adjusted for an array of potential confounders including age, ethnicity, education, smoking, congestive heart failure, cognitive and depressive status test scores, number of diseases, ankle-arm blood pressure, and diuretic use without history of hypertension or congestive heart failure, but not disability, ${ }^{23}$ therefore, it is not clear whether the nursing home placement risk predicted by frailty was independent of disability. Another possibility is cognitive impairment including dementia, which can lead to disability and dependence. ${ }^{36}$ Frailty has been shown to be associated with worse cognitive functions and to predict incident mild cognitive impairment and dementia, ${ }^{14,37}$ and adding cognitive impairment to frailty defined by physical components improved predictive validity for adverse outcomes. ${ }^{38}$ Furthermore, cognition has recently been considered as a part of frailty, ${ }^{37}$ and a new entity, cognitive frailty, has been proposed by an international consensus group as a heterogeneous clinical manifestation characterized by coexistence of physical frailty and cognitive impairment without concurrent dementia. ${ }^{32}$ Future research focusing on cause-specific nursing home placement may contribute to a further understanding of the associations between frailty and nursing home placement.

Mounting evidence shows that frailty generally deteriorates with age ${ }^{15}$ but also has the potential to be modified by appropriate interventions. ${ }^{7,17}$ Physical exercise may be a promising strategy to prevent or treat frailty ${ }^{39}$ In particular, aerobic and resistance exercise can be expected to address most of the core physical frailty components, such as weakness, slow gait speed, low physical activity, or sarcopenia. ${ }^{39}$ Previous interventional trials have shown that physical exercise improved muscle strength, gait speed, and physical performance. ${ }^{40-44}$ Furthermore, a 12 -month multifactorial interdisciplinary intervention, including tailored exercise programs, was conducted in frail community-dwelling older adults aged 70 or older in Australia and decreased the prevalence of frailty compared with the usual care group. ${ }^{45}$ It is of note that the adherence to the exercise programs was high and adverse events were not reported in most of the trials. ${ }^{39}$ These findings suggest that, potentially, physical exercise can prevent or reverse frailty and may lead to decreased risks of nursing home placement in older adults.

The findings must be interpreted with caution. Unadjusted ORs had to be used for the present 
meta-analysis because of limited data on adjusted effect measures available, therefore the findings may have been affected by important confounding factors, such as age, gender, education, or socioeconomic status. Future research should address the independent association between frailty and nursing home placement by controlling these factors.

Another potential limitation is that only 1 investigator conducted this study. It would have been theoretically more appropriate if 2 independent investigators had been involved in some processes of the systematic review. It is also of note that various types of frailty definitions were used by the included studies, and therefore different aspects of frailty may have been captured. Inconsistent use of frailty criteria by the included studies as well as other factors, including age, gender, or socioeconomic status may have contributed to moderate heterogeneity across the studies.

One of the major strengths of this review is that this is, to the best of my knowledge, the first meta-analysis on frailty as a predictor of nursing home placement. In addition to the metaanalysis, distinctive features of the current study include a more extensive search strategy and assessment of heterogeneity and publication bias across the included studies. Furthermore, additional data were obtained by contacting a corresponding author and were incorporated into the meta-analysis.

\section{Conclusion}

In summary, although it should be noted that the number of participants who were admitted to nursing homes were fairly small in all the studies, this systematic review and meta-analysis suggest that frailty may be a significant predictor of nursing home placement among community-dwelling older adults.

\section{CONFLICT OF INTERESTS}

None declared. 


\section{REFERENCES}

1. Ribbe MW, Ljunggren $\mathrm{G}$, Steel $\mathrm{K}$, et al. Nursing homes in 10 nations: a comparison between countries and settings. Age Ageing 1997;26 Suppl 2:3-12.

2. Ellis JM. Psychological transition into a residential care facility: older people's experiences. J Adv Nurs 2010;66(5):1159-1168.

3. Drageset J, Natvig GK, Eide GE, et al. Differences in health-related quality of life between older nursing home residents without cognitive impairment and the general population of Norway. J Clin Nurs 2008;17(9):1227-1236.

4. Centers for Disease Control and Prevention, Long-Term Care Services in the United States: 2013 Overview. 2013; http://www.cdc.gov/nchs/data/nsltcp/long_term_care_services_2013.pdf. Accessed 20th March, 2016.

5. Luppa M, Luck T, Weyerer S, et al. Prediction of institutionalization in the elderly. A systematic review. Age Ageing 2010;39(1):31-38.

6. Fried LP, Tangen CM, Walston J, et al. Frailty in older adults: evidence for a phenotype. J Gerontol A Biol Sci Med Sci 2001;56(3):M146-156.

7. $\quad$ Clegg A, Young J, Iliffe S, et al. Frailty in elderly people. Lancet 2013;381(9868):752-762.

8. Morley JE, Vellas B, van Kan GA, et al. Frailty consensus: a call to action. J Am Med Dir Assoc 2013;14(6):392-397.

9. Kojima G. Frailty as a predictor of hospitalisation among community-dwelling older people: a systematic review and meta-analysis. J Epidemiol Community Health 2016 (epub ahead of print).

10. Kojima G, Iliffe S, Jivraj S, et al. Association between frailty and quality of life among community-dwelling older people: a systematic review and meta-analysis. $J$ Epidemiol Community Health 2016 (epub ahead of print).

11. Kojima G, Iliffe S, Morris RW, et al. Frailty predicts trajectories of quality of life over time among British community-dwelling older people. Qual Life Res 2016 (epub ahead of print).

12. Kojima G, Kendrick D, Skelton DA, et al. Frailty predicts short-term incidence of future falls among British community-dwelling older people: a prospective cohort study nested within a randomised controlled trial. BMC Geriatr 2015;15:155.

13. Kojima G. Frailty as a predictor of future falls among community-dwelling older People: a systematic review and meta-analysis. J Am Med Dir Assoc 2015;16(12):1027-1033.

14. Kojima G, Taniguchi Y, Iliffe S, et al. Frailty as a predictor of Alzheimer's disease, vascular dementia, and all dementia among community-dwelling older people: a systematic review and meta-analysis (in press). J Am Med Dir Assoc 2016.

15. Collard RM, Boter H, Schoevers RA, et al. Prevalence of frailty in communitydwelling older persons: a systematic review. J Am Geriatr Soc 2012;60(8):1487-1492.

16. Kojima G. Prevalence of frailty in nursing homes: a systematic review and metaanalysis. J Am Med Dir Assoc 2015;16(11):940-945.

17. Lee JS, Auyeung TW, Leung J, et al. Transitions in frailty states among communityliving older adults and their associated factors. J Am Med Dir Assoc 2014;15(4):281286.

18. Bilotta $\mathrm{C}$, Nicolini $\mathrm{P}$, Case A, et al. Frailty syndrome diagnosed according to the Study of Osteoporotic Fractures (SOF) criteria and adverse health outcomes among community-dwelling older outpatients in Italy. A one-year prospective cohort study. Arch Gerontol Geriatr 2012;54(2):e23-28.

19. Drubbel I, de Wit NJ, Bleijenberg N, et al. Prediction of adverse health outcomes in 
older people using a frailty index based on routine primary care data. J Gerontol A Biol Sci Med Sci 2013;68(3):301-308.

20. Forti P, Rietti E, Pisacane N, et al. A comparison of frailty indexes for prediction of adverse health outcomes in an elderly cohort. Arch Gerontol Geriatr 2012;54(1):1620.

21. Coelho T, Paul C, Gobbens RJ, et al. Frailty as a predictor of short-term adverse outcomes. PeerJ 2015;3:e1121.

22. Rockwood K, Mitnitski A, Song X, et al. Long-term risks of death and institutionalization of elderly people in relation to deficit accumulation at age 70. $J$ Am Geriatr Soc 2006;54(6):975-979.

23. Bandeen-Roche K, Xue QL, Ferrucci L, et al. Phenotype of frailty: characterization in the women's health and aging studies. J Gerontol A Biol Sci Med Sci 2006;61(3):262266.

24. Jones D, Song X, Mitnitski A, et al. Evaluation of a frailty index based on a comprehensive geriatric assessment in a population based study of elderly Canadians. Aging Clin Exp Res 2005;17(6):465-471.

25. Wang SY, Shamliyan TA, Talley KM, et al. Not just specific diseases: systematic review of the association of geriatric syndromes with hospitalization or nursing home admission. Arch Gerontol Geriatr 2013;57(1):16-26.

26. Stroup DF, Berlin JA, Morton SC, et al. Meta-analysis of observational studies in epidemiology: a proposal for reporting. Meta-analysis Of Observational Studies in Epidemiology (MOOSE) group. JAMA 2000;283(15):2008-2012.

27. Wells GA, Shea D, O'Connell D, et al. The Newcastle-Ottawa Scale (NOS) for assessing the quality of nonrandomised studies in meta-analyses.

http://www.ohri.ca/programs/clinical_epidemiology/oxford.asp. Accessed 20th August, 2015.

28. Higgins JP, Thompson SG, Deeks JJ, et al. Measuring inconsistency in meta-analyses. BMJ 2003;327(7414):557-560.

29. Ensrud KE, Ewing SK, Taylor BC, et al. Comparison of 2 frailty indexes for prediction of falls, disability, fractures, and death in older women. Arch Intern Med 2008;168(4):382-389.

30. Rodriguez-Manas L, Feart C, Mann G, et al. Searching for an operational definition of frailty: a Delphi method based consensus statement: the frailty operative definitionconsensus conference project. J Gerontol A Biol Sci Med Sci 2013;68(1):62-67.

31. Walston J, Hadley EC, Ferrucci L, et al. Research agenda for frailty in older adults: toward a better understanding of physiology and etiology: summary from the American Geriatrics Society/National Institute on Aging Research Conference on Frailty in Older Adults. J Am Geriatr Soc 2006;54(6):991-1001.

32. Kelaiditi E, Cesari M, Canevelli M, et al. Cognitive frailty: rational and definition from an (I.A.N.A./I.A.G.G.) international consensus group. J Nutr Health Aging 2013;17(9):726-734.

33. Roppolo M, Mulasso A, Gobbens RJ, et al. A comparison between uni- and multidimensional frailty measures: prevalence, functional status, and relationships with disability. Clin Interv Aging 2015;10:1669-1678.

34. Rockwood K, Andrew M, Mitnitski A. A comparison of two approaches to measuring frailty in elderly people. J Gerontol A Biol Sci Med Sci 2007;62(7):738-743.

35. Vermeulen J, Neyens JC, van Rossum E, et al. Predicting ADL disability in community-dwelling elderly people using physical frailty indicators: a systematic review. BMC Geriatr 2011;11:33.

36. Prince M, Bryce R, Albanese E, et al. The global prevalence of dementia: a systematic 
review and metaanalysis. Alzheimers Dement 2013;9(1):63-75 e62.

37. Panza F, Solfrizzi V, Frisardi V, et al. Different models of frailty in predementia and dementia syndromes. J Nutr Health Aging 2011;15(8):711-719.

38. Avila-Funes JA, Amieva H, Barberger-Gateau P, et al. Cognitive impairment improves the predictive validity of the phenotype of frailty for adverse health outcomes: the three-city study. J Am Geriatr Soc 2009;57(3):453-461.

39. Aguirre LE, Villareal DT. Physical Exercise as Therapy for Frailty. Nestle Nutr Inst Workshop Ser 2015;83:83-92.

40. Latham NK, Bennett DA, Stretton CM, et al. Systematic review of progressive resistance strength training in older adults. J Gerontol A Biol Sci Med Sci 2004;59(1):48-61.

41. Liu CJ, Latham NK. Progressive resistance strength training for improving physical function in older adults. Cochrane Database Syst Rev 2009(3):CD002759.

42. Fiatarone MA, O'Neill EF, Ryan ND, et al. Exercise training and nutritional supplementation for physical frailty in very elderly people. $N$ Engl J Med 1994;330(25):1769-1775.

43. Frimel TN, Sinacore DR, Villareal DT. Exercise attenuates the weight-loss-induced reduction in muscle mass in frail obese older adults. Med Sci Sports Exerc 2008;40(7):1213-1219.

44. Fairhall N, Sherrington C, Lord SR, et al. Effect of a multifactorial, interdisciplinary intervention on risk factors for falls and fall rate in frail older people: a randomised controlled trial. Age Ageing 2014;43(5):616-622.

45. Cameron ID, Fairhall N, Langron C, et al. A multifactorial interdisciplinary intervention reduces frailty in older people: randomized trial. BMC Med 2013;11:65.

Figure 1. Flow chart of systematic literature review

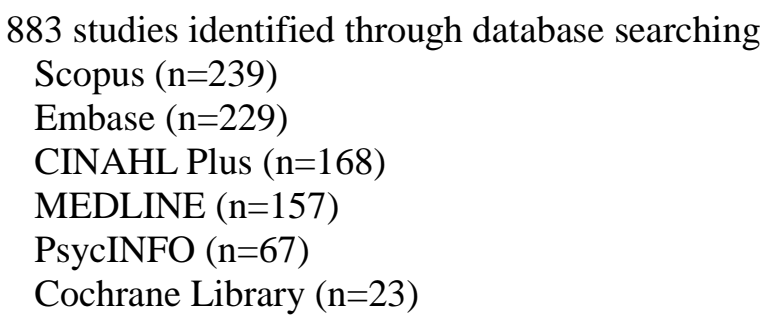

2 additional studies identified through other sources

442 studies screened for titles and abstracts

443 duplicated studies excluded

Total of 885 studies identified

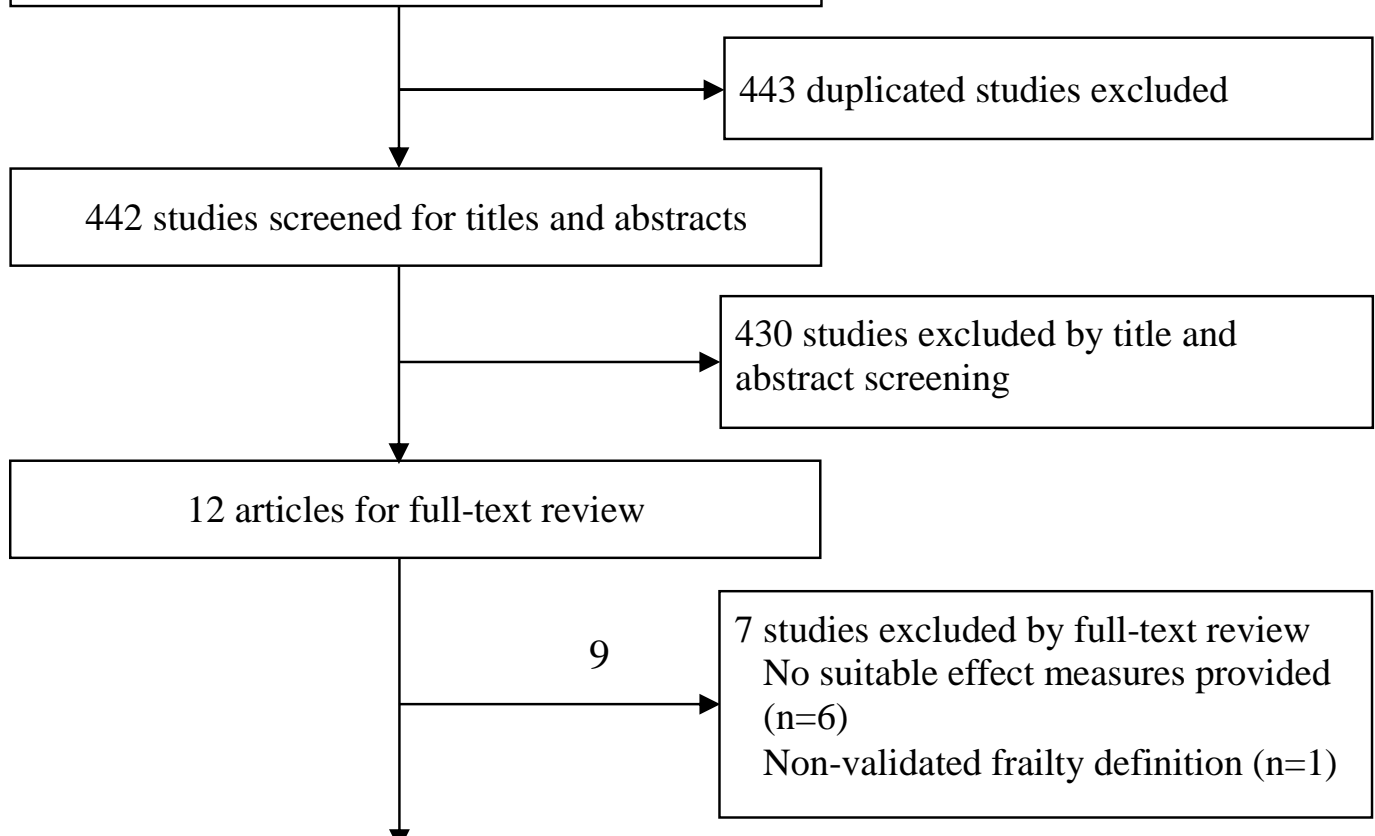


Figure 2. Forest plots of nursing home placement risk according to frailty.

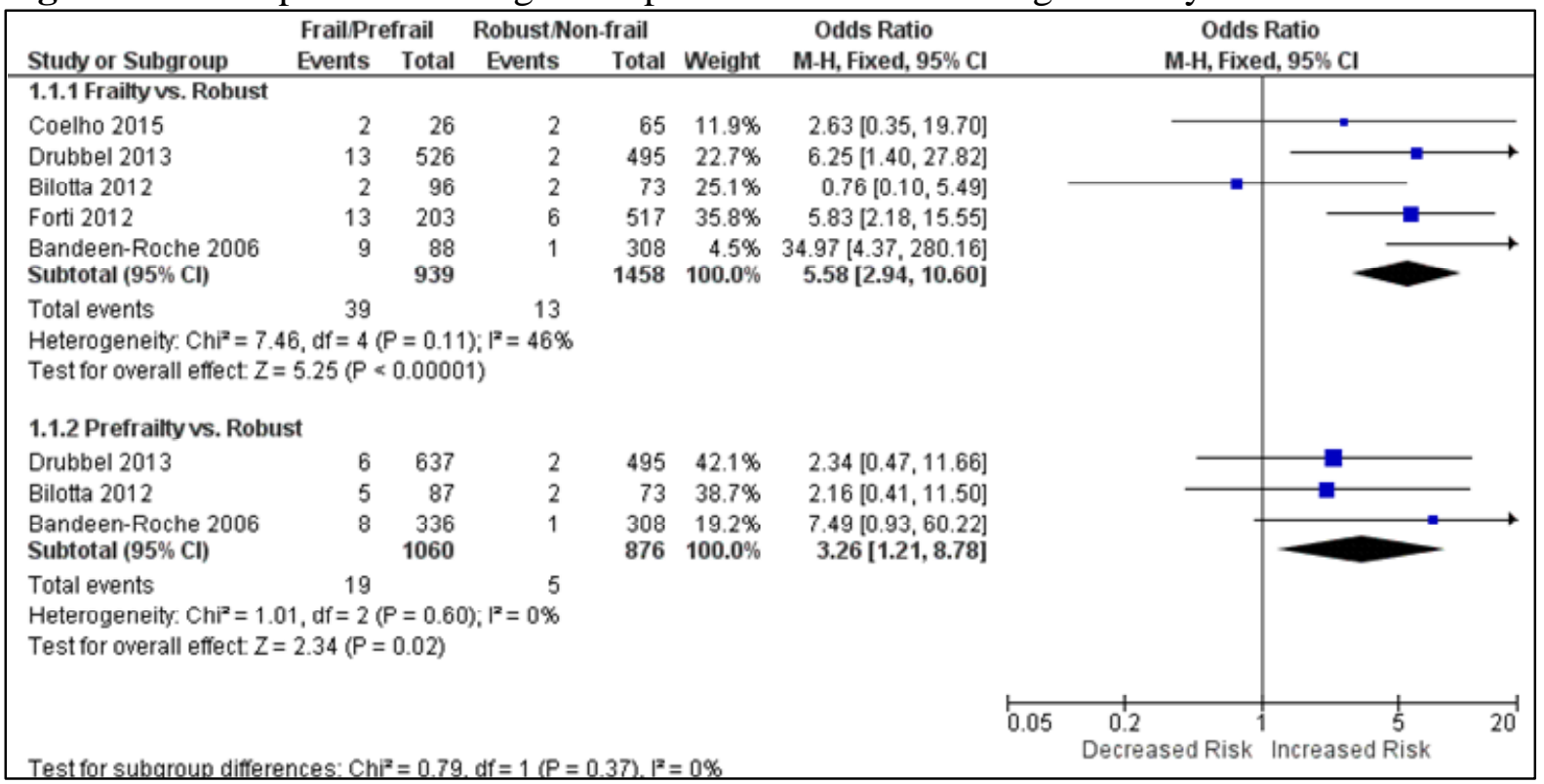


Figure 3 Funnel plots for nursing home placement risk according to frailty and prefrailty (A: studies with OR for frailty, B studies with OR for prefrailty)
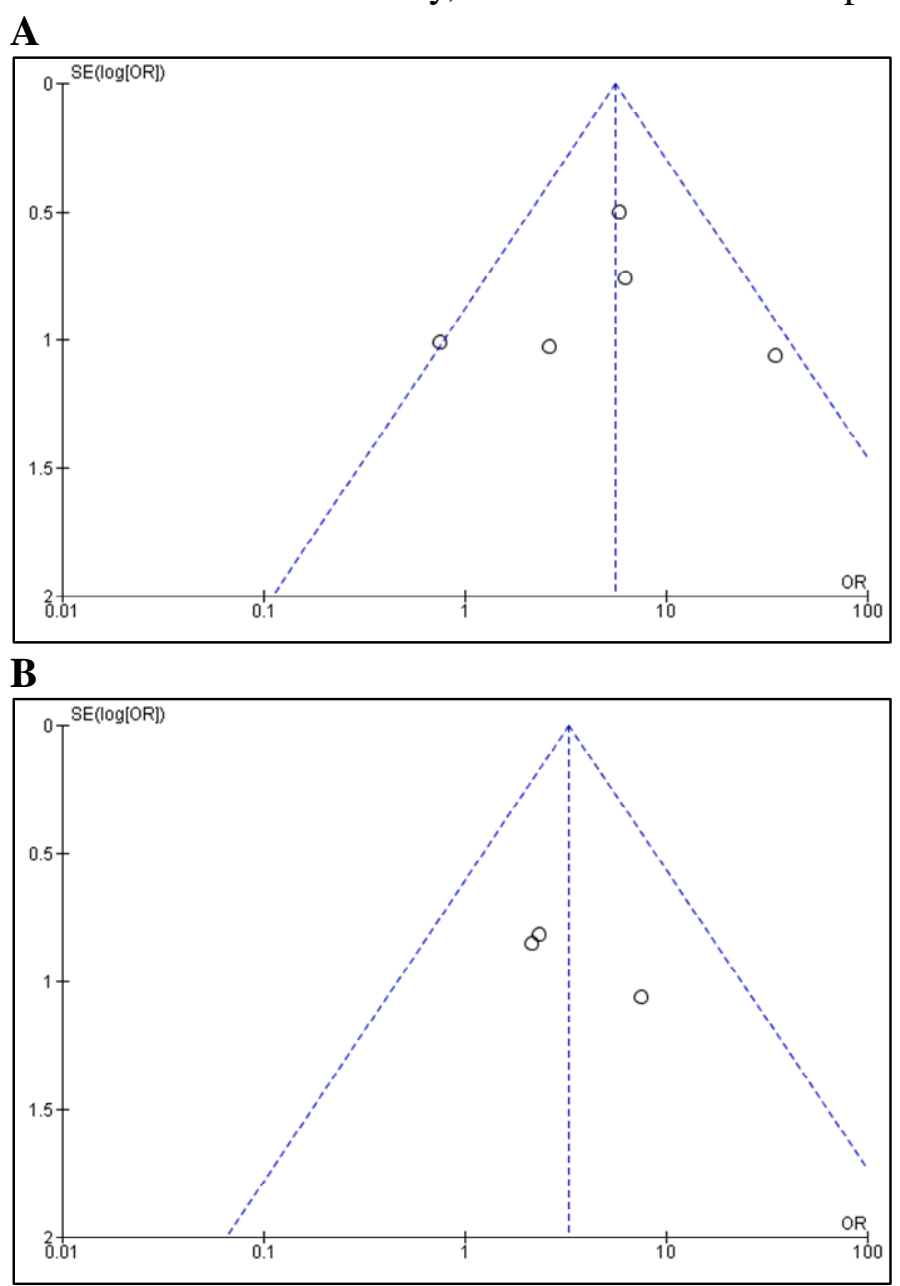
Table 1. Summary of included studies on frailty and nursing home placement among community-dwelling older adults.

\begin{tabular}{|c|c|c|c|c|c|c|c|c|c|}
\hline Author/Study & Year & Location & $\begin{array}{l}\text { Sample } \\
\text { size }\end{array}$ & $\begin{array}{c}\text { Female } \\
(\%)\end{array}$ & $\begin{array}{c}\text { Age } \\
\text { (range) }\end{array}$ & $\begin{array}{l}\text { Frailty } \\
\text { criteria }\end{array}$ & Effect measure & $\begin{array}{c}\text { Follow-up } \\
\text { period }\end{array}$ & NOS \\
\hline Coelho et al. ${ }^{21}$ & 2015 & Portugal & 95 & $67.4 \%$ & $\begin{array}{c}78.5 \\
(>65)\end{array}$ & mCHS & $\mathrm{cOR}$ & 10 months & $5 / 9$ \\
\hline Drubbel et al. ${ }^{19}$ & 2013 & Netherlands & 1679 & $58.8 \%$ & $\begin{array}{c}73^{\mathrm{a}} \\
(62-86)\end{array}$ & FI & $\mathrm{cOR}$ & 2 years & $6 / 9$ \\
\hline Bilotta et al. ${ }^{18}$ & 2012 & Italy & 265 & $71.3 \%$ & $\begin{array}{c}81.5 \\
(" 65+")\end{array}$ & SOF & $\begin{array}{l}\mathrm{uOR} \\
\mathrm{cOR} \\
\end{array}$ & 1 year & $7 / 9$ \\
\hline $\begin{array}{l}\text { Forti et al. }{ }^{20} \\
\text { CSBA }\end{array}$ & 2012 & Italy & 739 & $55.4 \%$ & $\begin{array}{c}74.7 \\
(\geq 65)\end{array}$ & CSBA index & $\begin{array}{l}\mathrm{uOR} \\
\mathrm{cOR} \\
\end{array}$ & 4 years & $5 / 9$ \\
\hline $\begin{array}{l}\text { Bandeen-Roche et } \\
\text { al. }{ }^{23} \\
\text { WHAS }\end{array}$ & 2006 & US & 750 & $100 \%$ & $(70-79)$ & $\mathrm{mCHS}$ & $\begin{array}{l}\text { aHR } \\
\mathrm{cOR}\end{array}$ & 3 years & $6 / 9$ \\
\hline
\end{tabular}

${ }^{a}$ Median age, aHR: Adjusted hazard ratio, CSBA: Conselice Study of Brain Aging, FI:

Frailty Index, mCHS: Modified Cardiovascular Health Study frailty index (Fried's

phenotype), mSOF: Modified Study of Osteoporotic Fractures frailty index, NOS: Newcastle-

Ottawa scale for cohort studies, u/cOR: Unadjusted/Calculated odds ratio, WHAS: Women's

Health and Aging Studies 